\title{
Eye surgery in the elderly
}

This article was published in the following Dove Press journal:

Clinical Interventions in Aging

5 April 2016

Number of times this article has been viewed

\section{Dorota Raczyńska \\ Leopold Glasner \\ Ewelina Serkies-Minuth \\ Magdalena A Wujtewicz \\ Kamila Mitrosz}

Department of Ophthalmology,

Medical University of Gdańsk,

Gdańsk, Poland

\section{Video abstract}

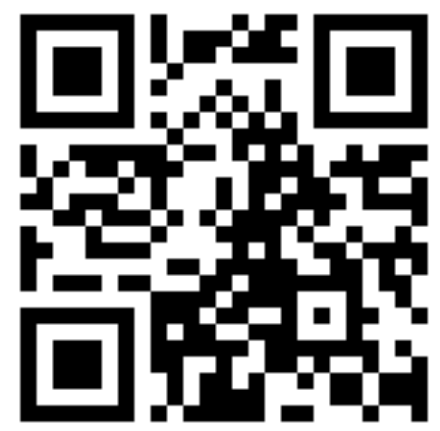

Point your SmartPhone at the code above. If you have a $O R$ code reader the video abstract will appear. Or use:

http://youtu.be//NwRV6it-PE
Correspondence: Dorota Raczyńska

Department of Ophthalmology, Medical University of Gdańsk, Smoluchowskiego

17, 80-952 Gdańsk, Poland

$\mathrm{Tel}+48583493580$

Email dorotaraczynska@gumed.edu.pl
Abstract: Extending life expectancy is a human achievement. It does however entail problems. Ophthalmic treatments are widely recognized as having a low risk of general complications. A classic example is cataract surgery, considered to be one of the safest and most frequently performed surgical procedures in the world. However, advanced age brings with it risks that should be considered before surgery. Eye operations, as with procedures on other organs, are largely dependent on the quality of surgical tissues. Therefore, the elderly are at increased risk of complications. Improved general health and postoperative follow-up with the use of noninvasive technologies such as optical coherence tomography translate into lower intraoperative risk and better postoperative prognosis. In this review, we discuss the impact of general health on operational prognosis, therapeutic problems, and technical difficulties which a surgeon and anesthesiologist may encounter in the process. We also consider new technology and strategies specifically aimed at treating eye conditions in the elderly.

Keywords: eye surgery, eye aging, anesthesiology in ophthalmology, cataract, glaucoma, vitrectomy, age-related macular degeneration

\section{Introduction}

Elderly people make up an increasingly growing proportion of patients in ophthalmologists' practices. On average, every third patient over the age of 65 is suffering from sight problems. ${ }^{1}$ The global number of older people (aged $\geq 60$ years) increased from $9.2 \%$ in 1990 to $11.7 \%$ in 2013 and will continue to grow as a proportion of the world population, reaching $21.1 \%$ by 2050 . Globally, this number is expected to more than double from 841 million people in 2013 to more than two billion in 2050. Presently, about two-thirds of the world's older people live in developing countries. ${ }^{2}$ Extended life expectancy results mainly from the promotion of a healthy life style, a reduced number of smokers, and greater involvement of patients in disease prevention, namely their participation in screening tests. ${ }^{3}$ Advances in medicine including access to high-tech diagnostic procedures, therapies, and surgical equipments also cannot be overestimated. Currently, the beginning of the old age is defined by the World Health Organization as 60 years of age.

\section{General health}

When performing surgery on the eyes in elderly patients, consideration must be given to the patients' general health status. If the person is in overall good health, eye surgery may not pose any major problems. Disorders that can significantly affect the condition of the eye and contribute to intraoperative complications include the following.

\section{Cardiovascular diseases}

Arterial hypertension

Hypertension (HTN)-associated risk in the elderly is several times higher than in younger patients. HTN and advanced age are the main risk factors of atherosclerosis. 
Arterial wall remodeling and endothelial damage and dysfunction induced by these factors result in progressive atherosclerosis with possible subsequent inflammation in the blood vessels.

In these patients, intraoperative fluctuations in arterial (particularly systolic) blood pressure and intraocular pressure (IOP) predispose them to arterial embolism and vein thrombosis. The same mechanism is observed in stroke. The risk of stroke in the elderly as a result of an increase in blood pressure is doubled in women and increased fourfold in men. ${ }^{4}$

\section{Heart valve disease}

Severe heart valve disease may preclude or delay eye surgery. In patients with artificial heart valves or atrial fibrillation, the decision to stop anticoagulants should be made by a cardiologist. In general, the risk of bleeding is higher for surgery of the orbit and eyelids and lower for surgery within the anterior segment of the eye. Most ophthalmic procedures (excluding plastic surgery, surgery of the orbit, and some vitrectomies) can be performed in these patients without the need to withdraw anticoagulants, including warfarin. ${ }^{5}$

\section{Respiratory disorders}

Elderly patients are at higher risk of postoperative respiratory complications, especially if the procedure is performed under general anesthesia. This applies mostly to patients who are unable to stay still during the surgical procedure, for example, patients with parkinsonism, bronchial asthma, and chronic obstructive pulmonary disease, or patients undergoing long and extensive procedures. The risk of postoperative respiratory distress is increased in elderly subjects, especially those with obesity, sleep apnea, and concomitant neuromuscular disorders, as well as when the action of the neuromuscular blocker used is not sufficiently reversed. All patients undergoing general anesthesia must be referred to the postanesthesia care unit, or when indicated, to the postoperative intensive care unit.

\section{Kidney and liver disorders}

Renal dysfunction is considered a major predictor of postoperative cardiovascular and pulmonary complications. ${ }^{6}$ Patients with end-stage renal disease undergoing longterm hemodialysis belong to a high-risk group (immune suppression, concomitant diseases). In such cases, the surgery must not delay dialysis. Otherwise, cardiovascular and respiratory complications may develop.

Patients with liver cirrhosis may be unsuitable for surgery because of impaired drug metabolism. Other consequences of liver failure including coagulopathies and thrombocytopenia may additionally complicate the procedure.

\section{Prostate gland disorders}

Benign prostatic hypertrophy is a common condition in men aged $>55$ years. Medicines such as $5 \alpha$-reductase inhibitors and $\alpha 1$-receptor-antagonists are associated with the "floppy iris syndrome", which may cause some difficulty during cataract surgery. ${ }^{7}$

\section{Endocrine disorders Diabetes mellitus}

Microangiopathy inherent to diabetes mellitus predisposes patients to the development of diabetic retinopathy and determines the options of surgical intervention. Poorly controlled blood glucose levels result in an abnormal immune response with an increased postoperative risk of infection, impaired healing, and inflammatory hyperreactivity.

Moreover, stress and anesthesia increase the production of inflammatory cytokines. According to the American Association of Clinical Endocrinologists and American Diabetes Association, the maximum acceptable fasting blood glucose level in patients suitable for surgery is $180 \mathrm{mg} / \mathrm{dL}$. It is also recommended that diabetic patients are scheduled as the first patients on operating lists. ${ }^{8}$

\section{Mental condition}

The interaction between the patient and treating physician is a key element while making therapeutic decisions, especially decisions about surgical treatment. Prior to making any decision, the patient and his/her family must receive reliable information about the inherent risks, possible reoperations, and the patient's responsibilities. Unwillingness to cooperate or rejection of the therapeutic process should be a clear signal to abandon the surgery. Obtaining written informed consent from the patient provides the surgeon with "a green light" to perform the best surgery. Additionally, especially in case of elderly patients with dementia or depression, the willingness to cooperate must be shown by the patient's family. This is of particular importance after the patient is discharged from hospital and requires extreme hygiene of the operated eye. It is recognized that awake surgery is better tolerated by mature patients who are more self-controlled and likely to cooperate with the surgeon. In contrast, younger patients are more frequently candidates for general anesthesia. It is interesting that in each category of patients, successful cataract surgery is associated with cognitive improvement and can even lead to the alleviation of depression. ${ }^{9}$ 


\section{Therapeutic solutions Cardiac pacemaker}

In general, in ophthalmic surgery, low-voltage coagulation is used for short periods of time. Where the eye surgery requires coagulation, the risk of pacemaker damage is negligible. Until now, no pacemaker manufacturer has reported damage to their device during ophthalmic surgery. ${ }^{10,11}$

\section{Anticoagulants and antibiotics}

According to reports from different sites, the use of anticoagulants is acceptable during cataract surgery and some types of glaucoma surgery. However, it is not recommended during vitrectomy. ${ }^{12}$ Katz et al having analyzed $>19,000$ cataract-operated patients aged $>50$ described the risk of taking aspirin or warfarin as minimal. ${ }^{13}$ Also, Carter et al did not report increased bleeding during cataract surgery in patients treated with anticoagulants. ${ }^{14}$

It is worth noticing that according to the American Heart Association, in most of the ophthalmic procedures, there is no need for systemic antibiotic prophylaxis.

\section{Topical intraoperative drug dosing}

Patient monitoring in cases of the intraoperative administration of drugs is particularly indicated in elderly patients in whom response to the medicines may be enhanced and lead to severe complications.

\section{Phenylephrine}

Phenylephrine used to dilate the pupil, especially when administered in concentrations $>10 \%$, may cause an increase in blood pressure in elderly patients and in extreme cases may lead to arrhythmia or even myocardial infarction. ${ }^{15}$

\section{Cyclopentolate}

Cyclopentolate, a mydriatic agent, especially in $>2 \%$ concentration, may impair functions of the nervous system and lead to psychoses and disorientation.

\section{Acetylcholine}

Acetylcholine used to constrict the pupil, along with pilocarpine, may cause a decrease in blood pressure, bradycardia, and bronchospasm.

\section{Anesthesia for eye surgery}

Anesthetic premedication involves the administration of commonly used short-acting sedatives or anxiolytic agents. However, caution is necessary in elderly patients as these agents may induce confusion.
Anesthetic management of patients, including the choice of a particular type of anesthesia for ophthalmic surgery, depends on the type of surgical procedure and the patient's condition. Most of the eye surgeries can be performed under local anesthesia. In order to improve patient safety, local anesthesia can be combined with monitored anesthesia care. In some cases, mild sedation is administered, and when it is required, due to the patient's condition or an extensive or painful procedure, general anesthesia is performed. Patients who are unable to stay still, mostly because of nervous system disorders accompanied by involuntary movements, and patients suffering from severe pain or dyspnea in supine position are candidates for general anesthesia. If there is an additional risk of pulmonary aspiration of gastric content, general anesthesia with endotracheal intubation is required.

The great majority of eye surgeons prefer local anesthesia as this enables patient cooperation. If a decision is made to perform awake surgery, sedation for the procedure must be appropriate. Only mild sedation is acceptable, and full contact with the patient must be maintained. If deep sedation is required, general anesthesia is safer. In each of these cases, monitoring of vital signs is mandatory.

As regards local anesthesia, it is administered by the surgeon, while monitored anesthesia care is provided by anesthesiologist. The aim of monitored anesthesia care during eye surgery is to respond immediately to any possible complications of regional anesthesia (eg, allergic reaction) including reflex bradycardia in the oculocardiac reflex, arrhythmia, and cardiac arrest following traction or compression of the eyeball, or sudden hypotension or HTN. As mentioned above, sedation should be avoided because sedative agents can impair communication with the patient. This may in turn lead to involuntary movement of the patient at the moment of weaker sedation, induce cough as a result of saliva flow, or cause respiratory depression that may sometimes require rapid restoration of the airway patency. Restoring airway patency during an ophthalmic procedure, either with simple measures or with breathing tubes, is a technically difficult maneuver because of the common procedure site of both the surgeon and the anesthesiologist. Upper airway obstruction may occur with either benzodiazepine or opioid use. An important contributing factor is the advanced age of the patient as in the elderly, the reaction to sedative agents may be unpredictable and is usually more pronounced than in younger patients.

If general anesthesia is indicated, to restore the airway patency, a laryngeal mask airway (LMA) can be used instead of endotracheal intubation. Both insertion and removal of 
the LMA cause much less injury and lower risk of adverse reflexes, which are particularly unwanted in patients with an eye pathology. ${ }^{16}$

Adverse reactions, which are sometimes dangerous, including tachycardia, HTN, and arrhythmia at the moment of insertion and removal of the breathing tube, occur considerably more frequently during endotracheal intubation than during LMA use. To assure safety in maintaining the LMA in its proper position throughout the whole procedure, the risk of LMA dislocation should be discussed with the surgeon. Anesthesia with the use of LMA as a breathing tube can be provided without the need for myorelaxant administration, which has the advantage of no risk of residual relaxation. Moreover, more rapid weaning from anesthesia enables a shorter stay for the patient in the operating theater.

However, if administration of a myorelaxant agent is necessary, short-acting myorelaxants should be used. The degree of muscle relaxation must be monitored, and the action of these agents must be reversed after the procedure.

The choice of anesthetic agents depends on the patient's condition, and the technique of providing anesthesia does not significantly differ from the commonly used methods. However, while providing anesthesia for eye surgery, some aspects should be taken into consideration.

In eye surgery, during intraocular procedures, ketamine should be avoided. Other anesthetics and opioids neither increase nor decrease IOP. ${ }^{17}$ At induction (intravenous or inhaled), blood pressure fluctuations should be avoided, and if endotracheal intubation is planned, the sympathetic response to stimulation of the larynx at intubation must be suppressed as strongly as possible. During anesthesia, both hypercapnia and hypoxemia must be avoided, as either of these conditions can increase IOP.${ }^{17}$ Maintaining normotension is recommended as HTN is associated with an increase in IOP. In cases of hypotension, especially in elderly patients, the compensatory mechanisms may be insufficient, and this applies not only to ophthalmic surgery but to any type of surgery. Extubation should be as smooth as possible to avoid cough.

As mentioned above, every time it is possible, LMA should be used instead of endotracheal intubation.

In retinal repair surgery, if nitrous oxide is used for anesthesia, the anesthesiologist must remember to stop the nitrous oxide administration and intensify anesthesia $\sim 15$ minutes before planned intraocular gas application (eg, SF6, C3F8). Nitrous oxide used concomitantly with intraocular gas may cause intraocular gas bubbles to expand and shrink.
This expansion and shrinkage may negate the effect of the surgery.

Another problem is anesthesia for emergency surgery, which is most frequently required in penetrating eye injuries. If possible, delaying the procedure until the patient has fasted is recommended. If it is not possible, rapid sequence induction should be conducted. If a decision is made to use succinylcholine, which can increase IOP by $5-10 \mathrm{mmHg}$ for 5-10 minutes, the risks and benefits of its use must be weighed. On one hand, there is a risk of more severe eye damage, and on the other hand, the risk of aspiration and hypoxemia. In studies involving hundreds of patients, no cases of prolapse of the eyeball have been reported as a result of succinylcholine use. ${ }^{18}$

An alternative option may be inhalational induction while maintaining the patient's own breathing.

Moreover, the anesthesiologist must be aware of the possibility of spreading the locally administered postretrobulbar anesthetics into the subarachnoid space and the risk of severe complication in the form of apnea. Furthermore, consideration should be given to the risk of untoward systemic effects of intraconjunctivally administered medications (eg, phenylephrine, ecothiopate).

An important element of the anesthesiologist's management is the patient's assessment for the presence of risk factors of postoperative nausea and vomiting. In patients with known risk of postoperative nausea and vomiting, all the procedures to prevent this complication must be followed. ${ }^{19}$ Opioids should be avoided in the postoperative care, especially as the need for their administration in eye surgery is uncommon. Vomiting in the postoperative period may totally negate the effect of the surgery.

\section{Surgical problems}

Undertaking eye surgery requires high precision, especially in geriatric patients in whom eye tissues are particularly fragile, for example, "rupturing" atrophic conjunctiva, atrophic floppy iris, and thinned, avascular, degenerated retina.

Problems can be encountered both in the anterior and posterior segment of the eye. During the whole process of treatment, every single element is important including preoperative management, the surgical procedure itself, and the postoperative period. Performing the surgery under conscious sedation seems to be a safer alternative in these patients. General anesthesia can more frequently result in postoperative malaise and vomiting, which is associated with the risk of complications including intraocular bleeding. 


\section{Anterior segment of the eye}

While planning an ophthalmic surgery, consideration must be given to every component of the eye. Not every disorder typical of the elderly affects the outcome of the surgery. Disorders of the protective structures of the eye including entropion, ectropion, lagophthalmos, ingrowing eyelashes, ciliary blepharitis, and meibomian gland may lead to subsequent complications..$^{20}$ The same applies to the disorders of the lacrimal system including a close up of a lacrimal punctum, lacrimal caruncle hypertrophy, and lacrimal drainage system obstruction or inflammation.

Disorders of the conjunctiva that must be taken into account include bacterial conjunctivitis and pterygium. Moreover, blink frequency should be considered. In the elderly, the blink frequency is usually lower. If symptoms of dry eye syndrome are already present before the surgery, a marked discomfort may be expected in the patient after the procedure. Reduced corneal transparency resulting from underlying keratopathy, dystrophy, or past inflammatory diseases will always pose some difficulty to the surgeon. The same is the case of excessive corneal thinning (eg, ulceration), ingrowth of the blood vessels into the corneal tissue, and decreased number of endothelial cells (with threatening postoperative corneal decompensation).

Most likely candidates for corneal transplantation in these groups of patients are those with Fuchs endothelial dystrophy. In elderly patients, transplant rejection is less common versus younger subjects. ${ }^{21}$

Disorders of the iris that make eye surgery more difficult include first of all floppy iris syndrome with signs and symptoms of neovascularization, atrophy, and poor mydriasis. A major problem is abnormal vascularization of the iris as well as anterior or posterior iris synechiae.

The surgical treatment of glaucoma should be estimated in the case of lack of cooperation with the patient, intolerance to drug treatment, and the necessity of high-pressure reduction. Most common and classic methods of glaucoma surgery such as shunts and trabeculectomy are associated with potentially hazardous outcomes. Rubeosis of the iris, frequently observed in neovascular glaucoma, is a relative contraindication for trabeculectomy because of fibrosis of the filtering shunt.

Therefore, several new approaches have been developed, for example, canaloplasty, the ex-press mini-shunt, and the trabectome device, which may be considered in treating the elderly.

Canaloplasty is a relatively new surgical non-penetrating procedure for restoring the natural outflow pathway of aqueous humor. It involves the dilatation of Schlem's canal with the use of microcatheter technology. Canaloplasty may not be as effective in reducing IOP as trabeculectomy. However, canaloplasty with cataract surgery achieves a higher IOP reduction than canaloplasty alone..$^{22}$

Since the introduction of the original Molteno implant 40 years ago, modifications of implants have been designed. An ex-press shunt, a stainless steel implant, has become an alternative and popular procedure. ${ }^{23}$ This is a glaucoma drainage device used to shunt aqueous from the anterior chamber into a subconjunctival reservoir that is created surgically. It is a relatively safe and effective procedure and provides satisfactory IOP control..$^{24}$

The trabectome device removes the trabecular meshwork and interior wall of Schlem's canal using an electrosurgical handpiece that disrupts the tissue. It has many advantages such as shorter surgery time, less intraoperative and postoperative complications, and a clear IOP-lowering effect. ${ }^{25}$ Moreover, the procedure can be performed with phacoemulsification.

Manual phacoemulsification with intraocular lens (IOL) implantation is the standard surgical procedure for removing cataract. This method utilizes high-energy ultrasound to fragment and emulsify the cataract. Surgical techniques in phacoemulsification are constantly developing to become safer and less invasive.

Phacoemulsification is mostly performed under topical anesthesia and requires small sutureless incisions in the eye. The small size of the incision allows low surgery-induced astigmatism, quick postoperative recovery, and complication reduction.

Heat transmission from the phacoemulsification needle to the cornea, which has a damaging effect on the corneal epithelium, has been minimized by modulating the energy into micro-pulses and micro-bursts. The protective silicone sleeve on the phacoemulsification needle also prevents corneal burn during surgery. ${ }^{26}$

Development in cataract surgery has also been related to IOL implants. Flexible and foldable IOLs can be implanted to the eye through a small incision in the cornea. A wide range of IOL implants, including toric IOLs correcting preoperative corneal astigmatism and multifocal IOLs expanding the range of clear vision, helps to achieve the desired postoperative refractive result.

Recently a femtosecond laser (FSL) has been introduced into phacoemulsification cataract surgery to open the lens capsule, fragment the cataract, and make corneal incisions, with corneal scarring being the only absolute contraindication 
for its use. ${ }^{27}$ FSL-assisted cataract surgery, compared to the conventional technique, requires less phacoemulsification energy and time, which may reduce the thermal injury to ocular tissues by ultrasound and corneal edema. ${ }^{27,28}$ Greater precision in stages of cataract surgery performed with FSL is suggested to improve visual and refractive outcomes in the short term. ${ }^{27}$ As the potential advantages of introducing FSL to cataract surgery are broad and yet not sufficiently evaluated, a randomized single masked trial has recently been designed to compare the safety and efficacy of FSLassisted cataract surgery in contrast to standard manual cataract surgery. ${ }^{29}$

Standard cataract surgery may also cause some problems. The procedure of cataract removal must be preceded with pupil dilation, but in the elderly, mydriasis is rather poor. In such cases, it may be necessary to administer adrenalin solution into the anterior chamber of the eye or even to use retractors.

Problems of capsulorhexis, which are caused by fragile capsule, are seen more frequently in eyes with mature cataract and pseudoexfoliation syndrome. ${ }^{30}$ A hypermature cataract can predispose to lens ectopia. In such cases, it is safer to remove the lens with a loop. ${ }^{31}$

\section{Posterior segment of the eye}

Abnormal lesions within the posterior segment of the eye are sometimes indications for surgery and are not necessarily associated with advanced age. However, age-induced degeneration and thinning of the tissues, for example, the sclera, may adversely affect performance of the surgery. An example of such a condition is scleral staphyloma, which makes scleral buckle surgery more difficult, or perforating scleromalacia affecting mostly elderly women with long history of rheumatoid arthritis. ${ }^{32}$

Refractory, recurrent corneal erosions in the elderly can be triggered by intraoperative corneal abrasions. Corneal epithelial abrasion is performed in case of prolonged vitrectomies when assessment of the fundus of the eye is limited (eg, corneal epithelial edema, keratopathy). Treatment of the recurrent corneal defects, especially in the elderly, is difficult and prolonged.

Surgical procedures within the posterior segment of the eye are usually longer and require more manipulation. As in the elderly, surgery under conscious sedation is preferred. The surgeon's experience is of great importance to avoid too long procedure. Elderly patients are more likely to suffer from osteoarthritis and neuralgia, which may pose a problem during prolonged surgery.
In order to decrease surgery time and eye trauma, smallgauge vitrectomy instrumentation (such as 23- and 25-gauge) has been introduced. The advantage of this technology includes less tissue manipulation, smaller sutureless incisions, and reduced inflammation and pain postoperatively with more rapid visual recovery. ${ }^{33}$ Small-gauge vitrectomy allows for overall faster patient recovery. ${ }^{34}$

New technologies such as microscope-integrated intraoperative optical coherence tomography allow us to observe microanatomical relationships during surgical repair of macula involving retinal detachment with pars plana vitrectomy. Intraoperative optical coherence tomography allows microanatomical structure of the macula to be examined and demonstrates features such as submacular fluid and macular flattening. ${ }^{35}$ Optical coherence tomography as a noninvasive real-time system can also play a crucial role in postoperative observation of the structure of macula in the elderly. It allows us to visualize cystoid macular edema, epiretinal membranes, and changes within photoreceptor layer. It assesses the retinal changes associated with visual outcome. ${ }^{36,37}$

There are also a few issues to keep in mind while operating on the elderly.

Geriatric patients with underlying atherosclerosis or diabetes have lower baseline retinal perfusion pressure with resultant high risk of ischemic events. ${ }^{38,39}$ These events are observed particularly in patients with advanced proliferative retinopathy. In such cases, successful surgery with improved architecture of the posterior segment of the eye does not result in vision improvement. During vitrectomy, it is even possible to observe a thrombus in vivo.

Another complication of arterial HTN may include suprachoroidal bleeding, especially in patients with concomitant glaucoma, a long eyeball, and vitreous outflow. ${ }^{40}$

Moreover, consideration should be given to the oculocardiac reflex, which may occur as a result of eyeball compression or traction to the extraocular muscles, for example, during episcleral filling implantation or strabismus correction. Resulting arrhythmias (sinus bradycardia, asystole, premature supra- and ventricular contractions) can be particularly dangerous for geriatric patients.

Last but not least, an important issue is the postoperative need for head positioning. This issue should be discussed prior to the surgery in order to avoid any misunderstanding and provide an optimal outcome of the surgery. If the patient is unable to maintain a body position recommended by the surgeon (eg, during vitrectomy with peeling for macular hole repair), the chances for vision improvement are poorer. 
We should also mention the role of intravitreal injections and drug implants (designed for administration into the anterior chamber and intravitreally). Constantly improving methods of drug delivery are used in the treatment of diseases such as exudative age-related macular degeneration, diabetic macular edema, glaucoma, and uveitis.

\section{Summary}

The process of human aging is highly individualized. It is affected by both genetic and environmental factors (eg, nutrition, social status, lifestyle). The health of every organ of the body, including the eye, depends to a great extent on the general health of the person. However, this general health does not always determine the occurrence of such conditions like cataract, degenerative myopia, or retinal degeneration. Many conditions of the eye are preventable by controlling risk factors including inappropriate diet or smoking. People shape the quality of their old age throughout the course of their lives.

Eye surgery in the elderly is successful in the great majority of patients and is associated with relatively few complications. Surgical techniques are highly advanced, and the procedures are performed on the level of microsurgery. Finally, it must be emphasized that good cooperation between the patients, their family, and the treatment team is a key factor for successful outcome of the surgery and the healing process.

\section{Disclosure}

The authors report no conflicts of interest in this work.

\section{References}

1. Quillen DA. Common causes of vision loss in elderly patients. Am Fam Physician. 1999;60(1):99-108.

2. World Population Ageing 2013 (Report). United Nations. Available from: http://blog.world-statistics.org/2014/03/world-population-ageing-2013. html. Accessed December 1, 2015.

3. Central Statistical Office. Department of Demographic Studies and Labour Market. Material for the press conference 30.01.2014r. Available from: http://stat.gov.pl/cps/rde/xbcr/gus/L_podst_inf_o_rozwoju_dem_ pl_do_2013.pdf. Accessed October 1, 2015.

4. Chodorowski Z. Geriatria z podstawami gerontologii [Geriatrics with Gerontology]. 2008:94-96. ISBN:978-83-922961-3-3.

5. Dunn AS, Turpie AG. Perioperative management of patients receiving oral anticoagulants: a systematic review. Arch Intern Med. 2003;163: 901-908.

6. Fleisher LA, Beckman JA, Brown KA, et al. ACC/AHA 2007 guidelines on perioperative cardiovascular evaluation and care for noncardiac surgery. Circulation. 2007;116:1971-1996.

7. Manvikar S, Allen D. Cataract surgery management in patients taking tamsulosin: staged approach. J Cataract Refract Surg. 2006;32: 1611-1614.

8. Gayer S, Zuleta J. Perioperative management of the elderly undergoing eye surgery. Clin Geriatr Med. 2008;24(4):687-700.
9. Ishii K, Kabata T, Oshika T. The impact of cataract surgery on cognitive impairment and depressive mental status in elderly patients. Am JOphthalmol. 2008;146(3):404-409.

10. Bayes J. A survey of ophthalmic anesthetists on managing pacemakers and implanted cardiac defibrillators. Anesthesia. 2006;103: 1615-1616.

11. Stoller GL. Ophthalmic surgery and the implantable cardioverter defibrillator. Arch Ophthalmol. 2006;124:123-125.

12. Charles S, Rosenfeld PJ, Gayer S. Medical consequences of stopping anticoagulant therapy before intraocular surgery or intravitreal injections. Retina. 2007;27:813-815.

13. Katz J, Feldman MA, Bass EB, et al. Risks and benefits of anticoagulant and antiplatelet medication use before cataract surgery. Ophthalmology. 2003;110:1784-1788.

14. Carter K, Miller KM. Phacoemulsification and lens implantation in patients treated with aspirin or warfarin. J Cataract Refract Surg. 1998; 24:1361-1364.

15. Fraunfelder FW, Fraunfelder FT, Jensvold B. Adverse systemic effects from pledgets of topical ocular phenylephrine 10\%. Am J Ophthalmol. 2002;134:624-625.

16. Agrawal G, Agarwal M, Taneja S. A randomized comparative study of intraocular pressure and hemodynamic changes on insertion of proseal laryngeal mask airway and conventional tracheal intubation in pediatric patients. J Anaesthesiol Clin Pharmacol. 2012;28(3):326-329.

17. Aitkenhead AR, Moppett I, Thompson J. Smith \& Aitkenhead's Textbook of Anaesthesia. 6th ed. Elsevier; 2013:601-619.

18. Butterworth IV JF, Mackey DC, Wasnick JD. Morgan \& Mikhail's Clinical Anesthesiology. 5th ed. McGraw-Hill Education; 2013:759-771.

19. Gan TJ, Diemunsch P, Habib AS, et al; Society for Ambulatory Anesthesia. Consensus guidelines for the management of postoperative nausea and vomiting. Anesth Analg. 2014;118(1):85-113.

20. Liang Q, Dong Z, Wang N. Perioperative ocular surface evaluation and management in meibomian gland dysfunction patients undergoing cataract surgery. Chin J Ophthalmol. 2014;50(4):244-246.

21. Duman F. Indications and outcomes of corneal transplantation in geriatric patients. Am J Ophthalmol. 2013;156(3):600.e2-607.e2.

22. Matlach J, Klink T. Trabeculectomy versus canaloplasty. Ophthalmologe. 2015;112(4):325-331.

23. Freedman J. What is new after 40 years of glaucoma implants. J Glaucoma. 2010;19(8):504-508.

24. Gavrić M, Gabrić N, Jagić J, Cović A. Clinical experience with ex-press mini glaucoma shunt implantation. Coll Antropol. 2011; 35 Suppl 2:39-41.

25. Huang $\mathrm{P}$, Wang $\mathrm{H}, \mathrm{Wu} \mathrm{H}$, et al. Preliminary investigations on the safety and efficacy of trabectome. Zhonghua Yan Ke Za Zhi. 2015;51(2): 115-119.

26. Devgan U. Surgical techniques in phacoemulsification. Curr Opin Ophthalmol. 2007;18(1):19-22.

27. Nagy ZZ, McAlinden C. Femtosecond laser cataract surgery. Eye Vis (Lond). 2015;2:11.

28. Chen X, Xiao W, Ye S, Chen W, Liu Y. Efficacy and safety of femtosecond laser-assisted cataract surgery versus conventional phacoemulsification for cataract: a meta-analysis of randomized controlled trials. Sci Rep. 2015;5:13123.

29. Day AC, Burr JM, Bunce C, et al; FACT Group. Randomised, single-masked non-inferiority trial of femtosecond laser-assisted versus manual phacoemulsification cataract surgery for adults with visually significant cataract: the FACT trial protocol. BMJ Open. 2015;5(11): e010381.

30. Hampton Roy F, Benjamin L. Cataract Surgery. 2007:70-71. ISBN:978-1-4160-2969-4.

31. Kański J. Clinical Ophthalmology. 2009:391. ISBN:978-83-7609120-4.

32. Kański J. Clinical Ophthalmology. 2009:353. ISBN:978-83-7609120-4.

33. Thompson J. Advantages and limitations of small gauge vitrectomy. Surv Ophthalmol. 2011;56:162-172. 
34. Lakhanpal R, Humayun M, de Juan E Jr, et al. Outcomes of 140 consecutive cases of 25-gauge tranconjunctival surgery for posterior segment disease. Ophthalmology. 2005;112:817-824.

35. Toygar O, Riemann C. Intraoperative optical coherence tomography in macula involving rhegmatogenous retinal detachment repair with pars plana vitrectomy and perfluoron. Eye (Lond). 2016;30(1):23-30.

36. Delolme M, Dugas B, Nicot F, Muselier A, Bron A, CreuzotGarcher C. Anatomical and functional macular changes after rhegmatogenous retinal detachment with macula off. Am J Ophthalmol. 2012;153(1):128-136.
37. Bae SH, Hwang JS, Yu Hg. Comparative analysis of macular microstructure by spectral-domain optical coherence tomography before and after silicone oil removal. Retina. 2012;32(9):1874-1883.

38. Langham ME, Grebe R, Hopkins S, et al. Choroidal blood flow in diabetic retinopathy. Exp Eye Res. 1991;52:167-173.

39. Dallinger S, Findl O, Strenn K, et al. Age dependence of choroidal blood flow. J Am Geriatr Soc. 1998;46:484-487.

40. Kański J. Clinical Ophthalmology. 2009:377. ISBN:978-83-7609120-4.

\section{Publish your work in this journal}

Clinical Interventions in Aging is an international, peer-reviewed journal focusing on evidence-based reports on the value or lack thereof of treatments intended to prevent or delay the onset of maladaptive correlates of aging in human beings. This journal is indexed on PubMed Central, MedLine,
CAS, Scopus and the Elsevier Bibliographic databases. The manuscript management system is completely online and includes a very quick and fair peer-review system, which is all easy to use. Visit http://www.dovepress. com/testimonials.php to read real quotes from published authors. 